\title{
Electroencephalographic brain dynamics of memory encoding in emotionally arousing context
}

\section{Carlos Enrique Uribe*, Ana Garcia and Carlos Tomaz}

Laboratory of Neurosciences and Behavior, Department of Physiological Sciences, Institute of Biology, University of Brasilia, Brasilia, DF, Brazil

\section{Edited by:}

Antonella Gasbarri, University of

I'Aquila, Italy

Reviewed by:

Antonella Gasbarri, University of

I'Aquila, Italy

Patrizia Campolongo, Università degli

Studi di Roma La Sapienza, Italy

${ }^{*}$ Correspondence:

Carlos Enrique Uribe, Neurosciences and Behavior Laboratory, Physiological

Sciences Department, Institute of

Biology, University of Brasilia, Campus

Darcy Ribeiro, IB Block G, $1^{\circ}$ level,

Brasilia, DF 70910-900, Brazil.

e-mail:ceuribev@unb.br
Emotional content/context enhances declarative memory through modulation of encoding and retrieval mechanisms. At encoding, neurophysiological data have consistently demonstrated the subsequent memory effect in theta and gamma oscillations. Yet, the existing studies were focused on the emotional content effect and let the emotional context effect unexplored. We hypothesized that theta and gamma oscillations show higher evoked/induced activity during the encoding of visual stimuli when delivered in an emotionally arousing context. Twenty-five healthy volunteers underwent evoked potentials (EP) recordings using a 21 scalp electrodes montage. They attended to an audiovisual test of emotional declarative memory being randomly assigned to either emotionally arousing or neutral context. Visual stimulus presentation was used as the time-locking event. Grand-averages of the EP and evoked spectral perturbations were calculated for each volunteer. EP showed a higher negative deflection from 80 to $140 \mathrm{~ms}$ for the emotional condition. Such effect was observed over central, frontal and prefrontal locations bilaterally. Evoked theta power was higher in left parietal, central, frontal, and prefrontal electrodes from -50 to $300 \mathrm{~ms}$ in the emotional condition. Evoked gamma power was higher in the emotional condition with a spatial distribution that overlapped at some points with the theta topography. The early theta power increase could be related to expectancy induced by auditory information processing that facilitates visual encoding in emotional contexts. Together, our results suggest that declarative memory enhancement for both emotional content and emotional context are supported by similar neural mechanisms at encoding, and offer new evidence about the brain processing of relevant environmental stimuli.

Keywords: brain dynamics, declarative memory, emotionally arousing context, evoked potentials, evoked spectral perturbations

\section{INTRODUCTION}

Emotionally arousing events are better remembered than neutral ones (Dolan, 2002; LaBar and Cabeza, 2006; McGaugh, 2006). If someone is asked about what they were doing when they found out about the death of a loved relative, everyone remembers about the central fact (the notice), but also whom they were with, where, and what were they doing in that moment. Such clear evidence, from the everyday experience, demonstrates the effect of emotion over memory. Both the content of the information and the context in which the information is embedded enhance memory formation (LaBar and Cabeza, 2006; Barrett et al., 2007; Pastor et al., 2008). Controlled experiments in laboratory settings reinforce this idea and allow studying the mechanisms and neural structures involved in this phenomenon.

Functional neuroimaging and electroencephalographic (EEG) recordings during the encoding of the stimuli have revealed some brain structures and mechanisms that are critical for the episodic memory formation. Although some evidence do not agree, the consensus is that during the encoding of episodic memories the activity of left hemisphere is increased, especially at the medial temporal lobe, the parietal lobe, and the prefrontal cortex (Sederberg et al., 2003, 2007; Osipova et al., 2006).

Consistent evidence demonstrate that EEG theta and gamma oscillations are increased during the encoding of stimuli that will be remembered, if compared to stimuli that will be forgotten
(Sederberg et al., 2003; Axmacher et al., 2006; Osipova et al., 2006; Guderian et al., 2009; Duzel et al., 2010; Jutras and Buffalo, 2010). This is the so called subsequent memory effect. Alpha oscillations have been studied also, however, there is less consensus about their role during memory encoding (Klimesch, 1997, 1999; Klimesch et al., 2000, 2005). The evoked and induced activities measured by the event-related potentials technique have shown even less agreement about the time windows and the source of the activity (Hillyard and Anllo-Vento, 1998; Vogel and Luck, 2000; Herrmann and Knight, 2001; Schupp et al., 2007). Nevertheless, methodological variances such as the kind of stimuli, the tasks used, and the type of measures extracted from the raw data might account for such divergence (Klimesch et al., 2000; Herrmann and Knight, 2001).

Some studies explored the effect of emotion over encoding and demonstrated a privileged access to neural processing resources that could lead to better memory formation (Bradley et al., 2001; Foti et al., 2009; Weinberg and Hajcak, 2010). They focused on the effect of the emotional content of visual stimuli, and found that the evoked and induced activities are modulated by the characteristics of the pictures. In one study, the effect of the emotional context over the processing of visual stimuli was assessed by the event-related potentials (ERP) technique (Pastor et al., 2008). They found that neutral stimuli induced higher late positive potentials (400-700 ms) when presented within an emotional context when 
compared to a mixed context. However, in this study the emotional context was created by the same visual stimuli when they were presented in blocks of stimuli with the same emotional valence. Nevertheless, this study did not test if the context exerted any effect over memory formation (subsequent memory effect). Moreover, the analyses applied in the above mentioned studies explored the effects of emotion using only time-domain techniques. Meanwhile, time-frequency decompositions might better describe these effects.

Our group has extensively used an emotional memory test (Cahill and McGaugh, 1995) in which visual and auditory information is delivered simultaneously (Frank and Tomaz, 2000; Gasbarri et al., 2005, 2006; Uribe et al., 2008). With this methodology we demonstrated that the emotional content enhances the episodic memory formation. However, the contribution of visual and auditory information in the memory facilitation could not be differentiated. In the present study we modified this methodology in order to explore the processing of visual information within the emotional context created by the narrative (Gasbarri et al., 2006). For this purpose we employed EEG recordings during the presentation of an emotional memory test in which the narrative preceded, and was not concomitant with, the visual stimuli presentation. We analyzed the brain activity related to visual stimuli processing through timedomain and frequency-domain methods.

We hypothesize that the auditory emotional context will enhance the episodic memory for the visual stimuli. This enhancement will be achieved through mechanisms similar to those present in the processing of stimuli with emotional content. Additionally, we hypothesize that oscillatory activity measured by time-frequency decompositions will be better suited to analyze the brain activity related to encoding of stimuli.

\section{METHODOLOGY \\ SUBJECTS}

Twenty-five right-handed volunteers [age 23.3 (SEM=0.9); 12 female] were recruited through announcement in the campus. None had antecedents of neurologic or psychiatric illnesses, concomitant use of medications or acoustic impair. Beck Depression Inventory and Beck Anxiety Inventory were applied to each volunteer. Scores of every volunteer were within the range expected in the normal Brazilian population (Gorenstein and Andrade, 1996) and none met the exclusion criteria. Written informed consent was obtained from each volunteer prior to any procedure. They did not receive money or other incentive for their participation. Volunteers were naïve about the aims of the study; however, at the end of the procedure, researchers explained to them the real objectives of the study. The experimental protocol was approved by institutional ethics committee.

\section{EMOTIONAL MEMORY TEST}

A series of 11 slides accompanied by a narration were used as stimuli. Slide consisted in the same material used by Frank and Tomaz (2000). Two different narrations could accompany these slides, both of them narrated by the same person with an unemotional voice and with a similar grammatical construction in both versions. The narrative is the same for both versions during the first four slides (Phase 1) and differs between versions during the remaining seven slides (Phase 2 and 3). The second part of the narrative (Phase 2 and 3) has emotionally neutral content in the Neutral (N) version and emotionally arousing content in the Emotional (E) version. A detailed description of the test could be found in Frank and Tomaz (2000). Narratives were delivered through headphones. In order to avoid concurrent processing of visual and audio information, narratives were delivered during the fixation cross images between the slides. Because an ERP study requires a minimal number of trials, each slide was presented twice (Figure 1). After the slide presentation, subjects were asked to score the story using the Self Assessment Manikin (SAM; Bradley and Lang, 1994), for both valence and arousal dimensions. One week later, the volunteers returned and performed a retrieval task in which they had to write down all the information they could remember about the slides and the narrative presented during the past session.

\section{EEG RECORDINGS}

EEG data were recorded using a NeuronSpectrum 4EP system (Neurosoft, Russia) at a sampling rate of $1000 \mathrm{~Hz}$ and with a 0.5$75 \mathrm{~Hz}$ band-pass filter. Twenty-one scalp electrodes (10-20 system) referenced to linked mastoids recorded the cortical activity of each volunteer. Each local of the electrodes was cleaned and prepared by gently rubbing with an abrasive gel (Nuprep, Weaver and Company, USA). A conductive paste (Ten20, Weaver and Company, USA) fixed the electrodes and maintained all the impedances below $5 \mathrm{k} \Omega$ during the whole session.

\section{PROCEDURE}

Volunteers were received in the recording room. They read and signed the written informed consent and responded to a brief interview before responding to the two inventories. Slides were presented in a $17^{\prime}$ flat screen at approximately $60 \mathrm{~cm}$ from the subject. They sat comfortably in front of the screen while the electrodes and the

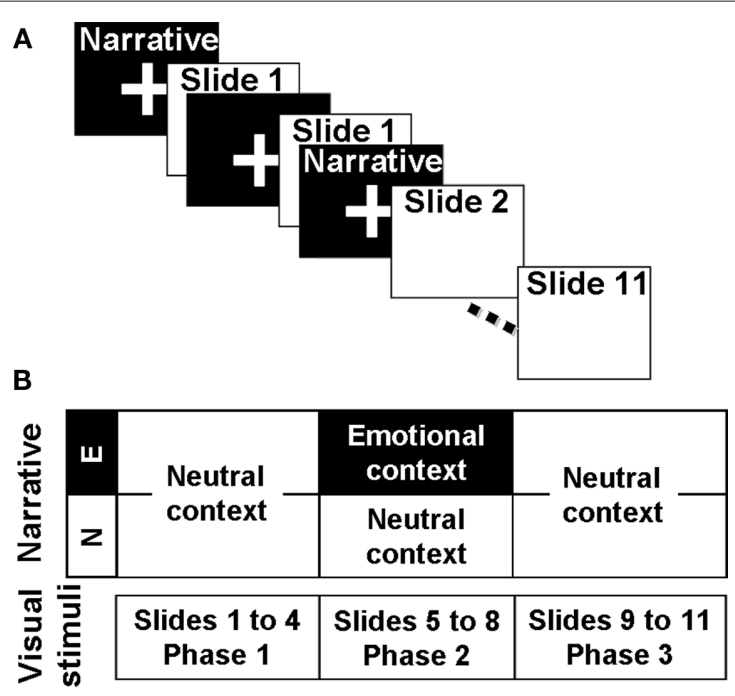

FIGURE 1 | Schematic representation of the experimental procedure. (A) Slide presentation sequence. Narrative was not concomitant with the slide presentation and preceded the image of the scene it describes. As shown, each slide was presented twice. (B) Auditory and visual material. Images were the same for both versions of the test and were grouped in three phases. The narrative that accompanied the Phase 2 was different for each version. 
headphones were installed. The researcher asked to stay as quite as possible in order to avoid artifacts and to attend to both slides and narrative as they attend to a TV show. After the slide presentation, volunteers responded to the SAM and left the room. One week after, they returned to perform the retrieval task. Luminance, audio volume and instructions were kept constant.

\section{DATA PROCESSING}

All data processing and analyses were performed using EEGLAB v9.0.0.0b a freely available open source toolbox (http://sccn.ucsd. edu/eeglab/) suited for EEG data management under Matlab ${ }^{\circledast}$ or Octave platform (Delorme and Makeig, 2004; Makeig et al., 2004).

\section{ICA DECOMPOSITION}

Standard channel coordinates of the BEM dipfit model were used for all the electrodes. ICA decomposition was performed using the Infomax (runica) algorithm (Bell and Sejnowski, 1995). Both Rest (Phase 1) and Test (Phase 2) periods were submitted to the same analysis as concatenated datasets. EEG recordings were decomposed in 21 independent components (IC). Eye blink and saccadic movements (from the Test period) were always represented by two or three IC which were selected and eliminated in both rest and test datasets. The back-projection of the remaining IC resulted in the "clean datasets" used for all the analyses.

\section{EVOKED POTENTIALS (EP) AND EVOKED SPECTRAL PERTURBATIONS (ESP)}

In some cases, differences in the mean average evoked activity (time domain) were not found, while differences in oscillatory activity (frequency domain) appeared (Klimesch et al., 2000; Makeig et al., 2004; Osipova et al., 2006).

Events were recorded in a separate channel during the Test period and then extracted to set the time 0 for each epoch. Mean baseline $(-1000$ to $0 \mathrm{~ms})$ was subtracted from each epoch. Several methods to reject bad epochs were applied to the 21 channels. All epochs with extreme values (higher than $|75| \mu V$ ), abnormal trend (slope higher than $50 \mu \mathrm{V} /$ epoch and $R^{2}$ higher than 0.3 ), improbable data (data beyond $4 \mathrm{SD}$ in a single channel or all channels), abnormal data distribution (any epoch with data distribution beyond $5 \mathrm{SD}$ ) or abnormal spectrum (higher than $\mathrm{I} 25 \mathrm{I} \mathrm{dB}$ in the $0-2 \mathrm{~Hz}$ range) were automatically rejected. The remaining epochs were used in the EP and ESP analyses. EP grand-average was calculated for each volunteer. ESP images were computed based on 10 trials of 3000 frames using three cycles at the lowest frequency and 250 cycles at the highest. Frequency resolution was set to $100 \log$ spaced points from 3 to $500 \mathrm{~Hz}$.

\section{STATISTICAL ANALYSES}

Electrophysiology and neuroimaging studies use the subsequent memory effect to point out which measures can be associated with encoding success (Sederberg et al., 2003; Osipova et al., 2006; Jutras and Buffalo, 2010; Nyhus and Curran, 2010). This can be done by comparing the brain activity at the encoding of an item that is successfully retrieved or recognized in a later session against the activity at the encoding of an item that is not retrieved/recognized. On the other hand, we classified the items based on the context they were embedded as previous studies did (Cahill and McGaugh, 1995; Frank and Tomaz, 2000; Gasbarri et al., 2005; Uribe et al., 2008).
Despite the present work did not select the items based on individual retrieval, the methodology is compatible with the subsequent memory effect.

Independent-samples t-tests compared age, arousal, and valence between groups. Retrieval scores were divided according to the phase the information belongs to. These scores were analyzed by a mixed design ANOVA with the factors Group (Neutral or Emotional; between-subjects) and Phase (1 and 2; within-subjects). Post hoc analyses were performed with independent-samples t-tests. Significance level for multiple comparisons was adjusted with Bonferroni method. Analyses of the EEG data (EP and ESP) were performed with the parametric statistical tools provided by the EEGLAB platform. Only data from Phase 1 and Phase 2 were considered, because they best represent baseline and experimental conditions. Results are expressed as mean (SEM).

\section{RESULTS}

\section{BEHAVIORAL DATA}

Mean age were not different between the Emotional (E) and Neutral (N) groups $[21.7(0.7)$ and 24.8 (1.6) respectively; $p=0.062]$. As expected, the mean valence score was lower and the mean arousal score was higher in the E group. Total retrieval, as well as Phase 1 and Phase 3 retrieval, was not different between groups. However, mean score of Phase 2 retrieval was higher in the E group (Table 1).

\section{EVOKED POTENTIALS}

Grand-average potential related to Phase 2 slides differed between groups around $120 \mathrm{~ms}$ (from 80 to $140 \mathrm{~ms}$ ) after the stimuli onset (Figure 2A). The anterior (frontal and central) electrodes registered a more negative potential in the E group during this time interval (Figure 2B). There were some spurious differences between groups at later times but they were not sustained over time and lasted no more than $10 \mathrm{~ms}$.

\section{OSCILLATORY ACTIVITY - EVENT-RELATED SPECTRAL PERTURBATIONS Theta band (4-7 Hz)}

Prefrontal (Fp1, Fpz, Fp2), frontal (F7, F3, Fz, F4, F8), central (C3, $\mathrm{Cz}, \mathrm{C} 4)$, and left parietal (P3, Pz) electrodes showed higher thetaband power in the E group than in the $\mathrm{N}$ group at the onset of the Phase 2 slides. In some cases, such differences were present even before the stimuli onset $(-50 \mathrm{~ms})$. Theta-band power remained higher after $300 \mathrm{~ms}$ from the stimuli onset in prefrontal, left frontal, left central, and midline parietal electrodes (Figure 3 ).

Table 1 | Mean (SEM) scores of behavioral data for each group.

\begin{tabular}{lccr}
\hline & Neutral & Emotional & $p$-value \\
\hline Valence & $5.2(0.4)$ & $2.3(0.4)$ & $<0.001$ \\
Arousal & $2.8(0.8)$ & $5(0.9)$ & 0.041 \\
RETRIEVAL & & & \\
Total & $15.2(2.1)$ & $12.6(0.9)$ & 0.138 \\
Phase 1 & $4.7(0.6)$ & $4.8(0.6)$ & 0.853 \\
Phase 2 & $2.5(0.5)$ & $6.2(0.5)$ & $<0.001$ \\
Phase 3 & $4.3(0.7)$ & $3.8(0.7)$ & 0.303
\end{tabular}



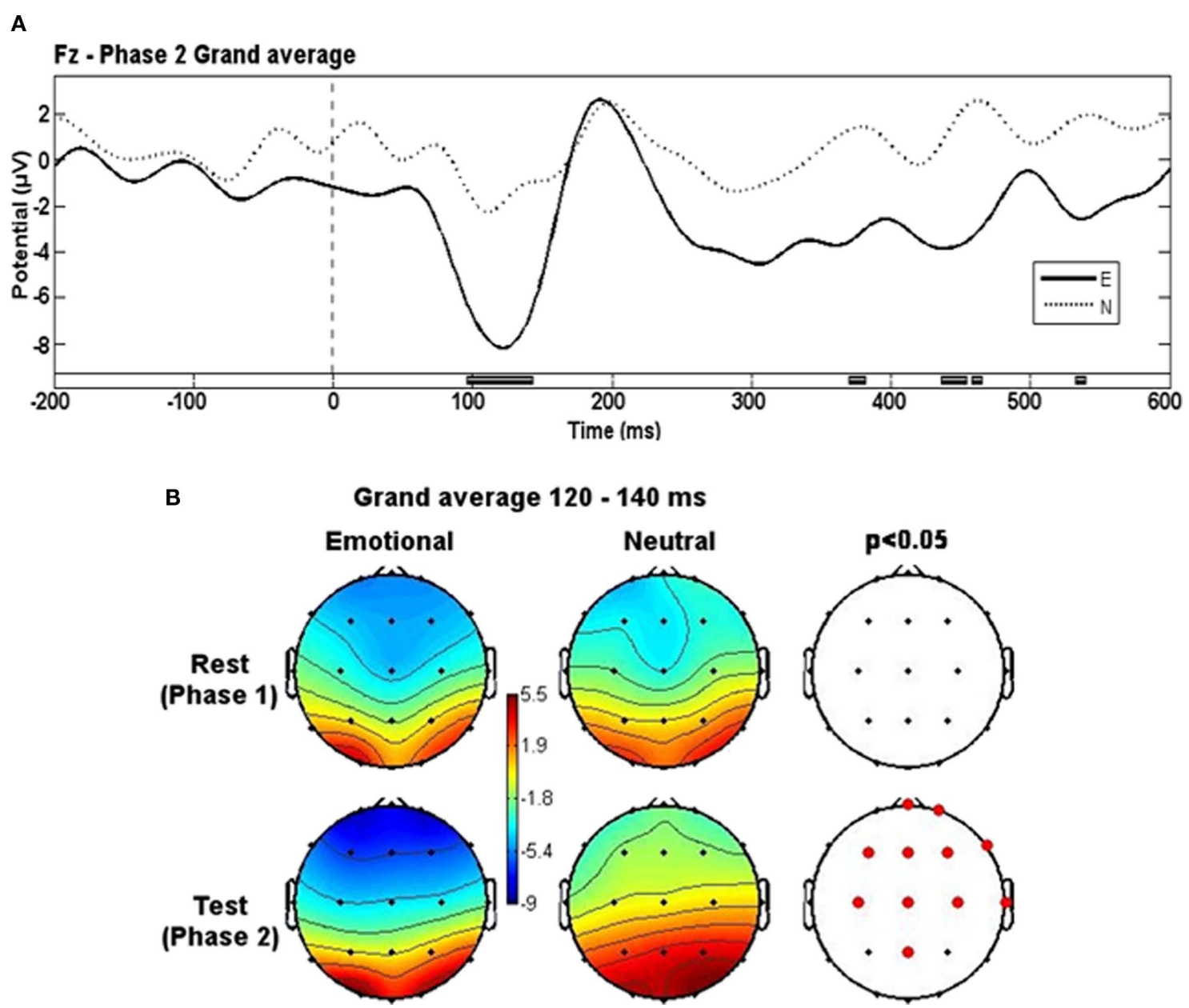

FIGURE 2 | Evoked potentials grand-averages. (A) Phase 2 grand-averages for $\mathrm{E}$ and $\mathrm{N}$ groups. Black blocks at bottom represent the time intervals where significant differences appeared. Low-pass filter of $20 \mathrm{~Hz}$. (B) Topographical representation of activity for each group in both phases at the $120-140 \mathrm{~ms}$ time window. Red dots represent electrode locations where significant differences appeared.

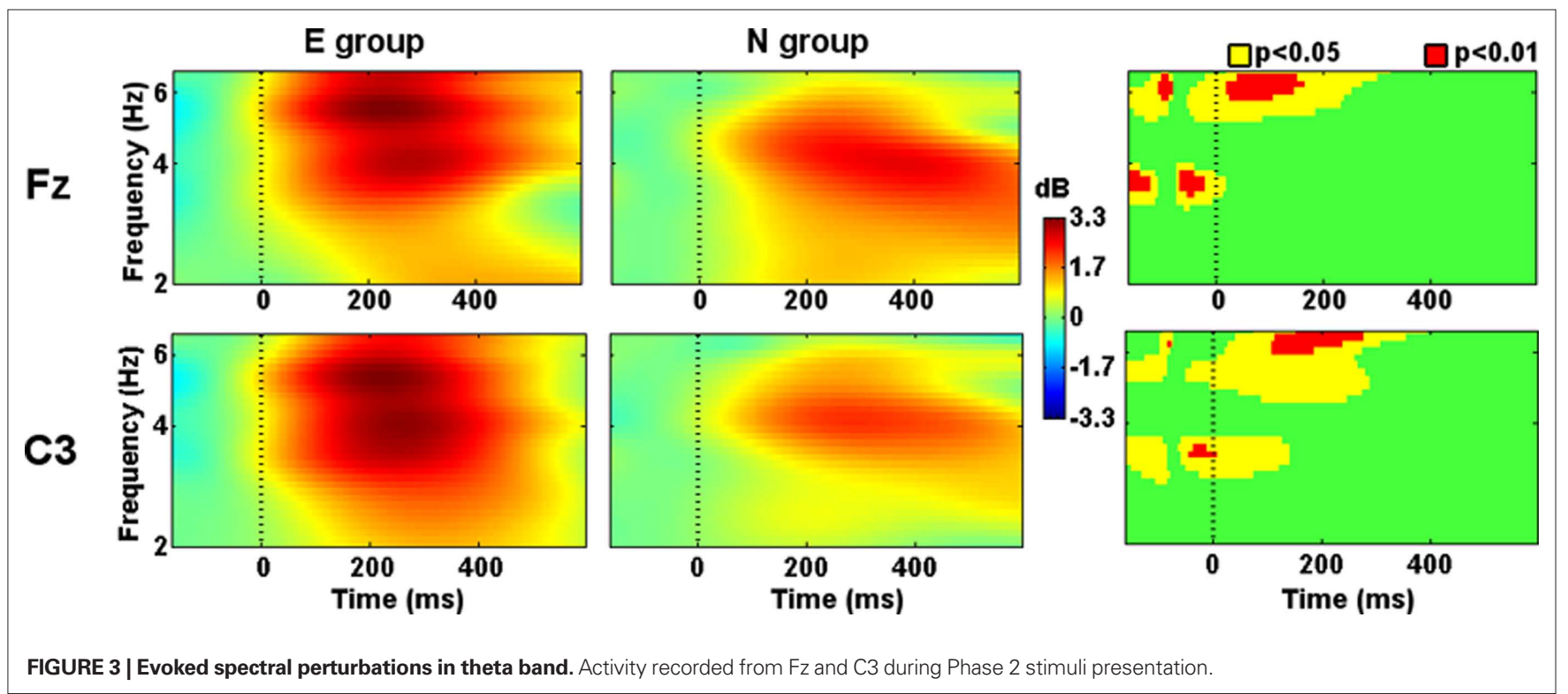




\section{Alpha band (7-13 Hz)}

Power within 10-12 Hz was higher in the E group than in the $\mathrm{N}$ group at right parietal (P4) scalp location from $-50 \mathrm{~ms}$ to $400 \mathrm{~ms}$ after the Phase 2 stimuli onset (Figure 4). This differential activity was not stationary and showed some changes. At higher latencies, the frequency where such differential activity appeared was faster.

\section{Gamma band (30-50 Hz)}

Since this frequency band is wide, we decided to split it in Gamma1 $(\leq 40 \mathrm{~Hz})$ and Gamma2 $(>40 \mathrm{~Hz})$ in order to facilitate the description and discussion of the results. Gammal showed higher activity in the $\mathrm{N}$ group from -50 to $400 \mathrm{~ms}$ after the Phase 2 stimuli onset in the T6 location (Figure 5). Around $300 \mathrm{~ms}$ left prefrontal (Fp1), right central (C4), left temporal (T3), and occipital locations (O1, $\mathrm{Oz}$, and $\mathrm{O} 2$ ) showed increased activity in the $\mathrm{N}$ group too. In $\mathrm{T} 3$ and C4 (Figure 5), such activity was sustained until $500 \mathrm{~ms}$ after the stimuli onset.

Gamma2 showed higher activity in the E group from 0 to $400 \mathrm{~ms}$ in the F7 location (Figure 5). From 300 to $400 \mathrm{~ms}$ left parietal (P3) showed higher activity in the E group and, right central (C4) electrode showed higher activity in the $\mathrm{N}$ group (Figure 5). From 400 to $500 \mathrm{~ms}$ right occipital (Oz, O2) and
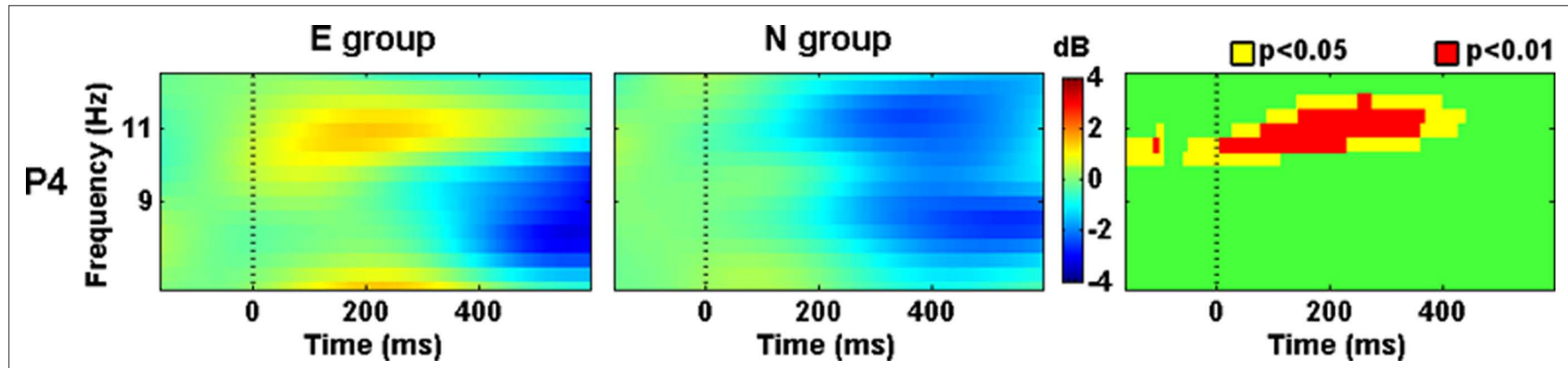

FIGURE 4 | Evoked spectral perturbations in alpha band. Activity recorded from P4 during Phase 2 stimuli presentation.
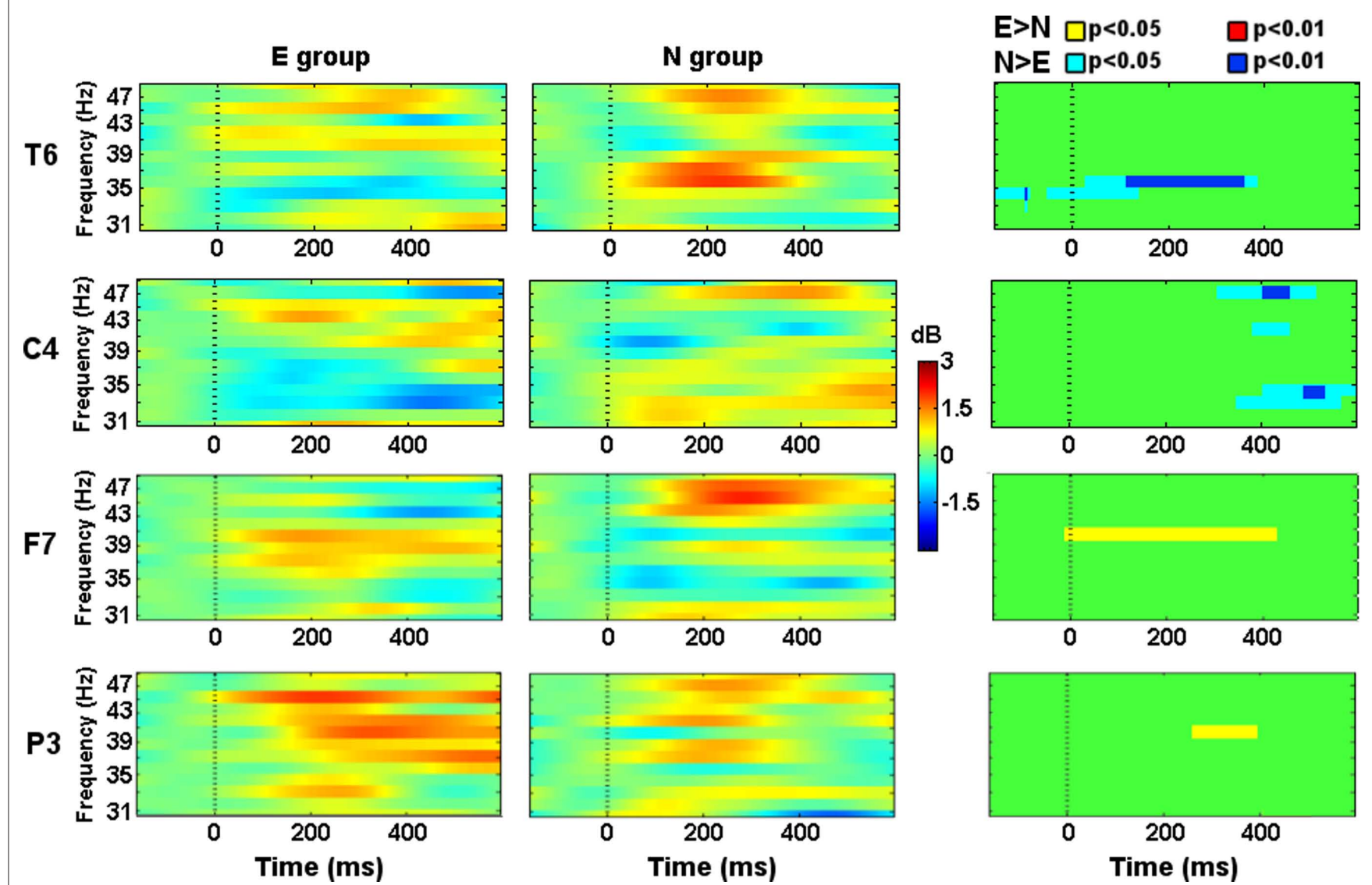

FIGURE 5 | Evoked spectral perturbations in gamma band. Activity recorded from T6, C4, F7, and P3 during Phase 2 stimuli presentation. 
parietal (P4) showed higher activity in the E group and, prefrontal (Fp1, Fpz, Fp2) electrodes showed higher activity in the $\mathrm{N}$ group.

\section{DISCUSSION}

This study explored the brain dynamics, through EP and ESP, during the encoding of visual stimuli in either emotionally arousing or neutral contexts.

\section{AROUSAL, VALENCE AND RETRIEVAL}

Behavioral data replicated previous results that demonstrated higher arousal and lower valence scores for the emotional version of this test (Cahill and McGaugh, 1995; Frank and Tomaz, 2000; Gasbarri et al., 2005; Uribe et al., 2008). These results ensure that each group was exposed to the same visual stimuli but in a diverse context. Hence, we can state that any difference in memory performance and in brain activity can be attributed to the emotional context.

Regarding retrieval, our results showed a memory enhancement for items related to the second phase. In line with previous studies (Cahill and McGaugh, 1995; Frank and Tomaz, 2000; Gasbarri et al., 2005), the effect over declarative memory is limited to Phase 2 of the emotional version of this test, in which emotion is delivered.

\section{EVOKED POTENTIALS}

Early EP (P1 and N1) have been related to sensory processes and attention (Luck et al., 1990; Hillyard and Anllo-Vento, 1998; Vogel and Luck, 2000). In both auditory and visual domains, it is assumed that these potentials are modulated by the physical characteristics of the stimuli and not by cognition. Some authors differentiate the attentional effect over each potential and suggest that $\mathrm{P} 1$ represents the facilitation of the sensory processing for stimuli presented at an already attended location (spatial selective). On the other hand, N1 might represent the orienting response toward task-relevant stimuli (object selective; Luck et al., 1990; Hillyard and Anllo-Vento, 1998; Herrmann and Knight, 2001; Beaucousin et al., 2011). However, some authors argue that N1 is also modulated by previous experience and by the semantic category of the stimuli configuring the called "initial visual categorization" phenomenon (Bentin and Golland, 2002).

Emotional stimuli (both appetitive and aversive) capture attention (Bradley et al., 2001), and attention is also voluntarily directed to them (Schupp et al., 2007). Several studies showed greater negativities in early time windows (N1) for emotional compared to neutral stimuli (Schupp et al., 2007; Foti et al., 2009; Weinberg and Hajcak, 2010) reflecting such preferential processing and the initial visual categorization phenomenon.

The results of the present study showed that emotional context modified the brain activity (deeper negativity) evoked from 80 to $140 \mathrm{~ms}$ after visual stimuli onset. Nevertheless, pictures in both versions of the test were the same. The differential processing of visual information can only be attributed to the emotional context created by the auditory information. This statement is in agreement with the initial visual categorization phenomenon if we consider that identical images can be interpreted differently depending on their context.
The attention effect over N1 commonly shows a posterior distribution (central, parietal, and occipital; Vogel and Luck, 2000; Weinberg and Hajcak, 2010), but anterior distribution (central and frontal) has been found too (Vogel and Luck, 2000; Töllner et al., 2009). In one study using simple or choice-reaction time to visual stimuli, the anterior N1 found was actually a brain potential related to pre-motor activity or preparatory processes (Vogel and Luck, 2000). The predictable timing of the pictures in the task was responsible, since an unpredictable onset of the stimuli suppressed the anterior N1. Although in the present study the visual stimuli onsets were predictable, subjects did not perform any motor response. Then, the results suggest that other preparatory processes (e.g., expectancy, arousal) might be increased by an emotional context.

Yet, another study considered the anterior N1 as the neural index of attention during modality shifting (Töllner et al., 2009). It demonstrated that the amplitude of the anterior N1 component was higher when the target modality (visual or tactile) changed between trials. It also ruled out any contribution of response-related factors. In the present study, the first of the two appearances of each picture was preceded by the narrative in both versions. If $\mathrm{N} 1$ is due only to modality shifting, differences between groups might not appear. If the modality shifting effect was present in one half of the stimuli, we can conclude that the emotional context boosted this effect. Interestingly, the anterior N1 of the modality shifting effect (Töllner et al., 2009) had a left lateralized distribution similar to the results of the present study.

\section{OSCILLATORY ACTIVITY}

Growing evidence demonstrates that modulation of the oscillatory brain activity is related to successful memory encoding (Klimesch et al., 1997b; Axmacher et al., 2006; Osipova et al., 2006; Jutras and Buffalo, 2010; Nyhus and Curran, 2010). The results of the present work are consistent with previous studies and demonstrated that changes in theta, alpha, and gamma bands enhance declarative memory.

\section{THETA OSCILLATIONS}

The amplitude of theta oscillations in the medial temporal lobe predicted the successful encoding of the stimuli (Klimesch et al., 1997b; Klimesch, 1999; Osipova et al., 2006). If the timing of stimuli onset is predictable, theta oscillations are higher during the encoding of remembered items even before the stimuli onset (Sederberg et al., 2003, 2007; Guderian et al., 2009). This pre-stimuli theta level seems to be an active process that acts independently and in synergy with other cognitive mechanisms that facilitate encoding such as the level of processing, novelty expectancy, spatial location, etc. (Guderian et al., 2009).

Theta evoked and induced activity over the left hemisphere was higher in the E group. This wide spread activity is coherent with its role of communicating functionally linked networks from remote locations (Buzsaki and Draguhn, 2004; McCartney et al., 2004; Axmacher et al., 2006; Klimesch et al., 2008; Duzel et al., 2010; Nyhus and Curran, 2010). The stimuli in the present study had a predictable timing and the results showed an increase in theta oscillations even before the stimuli onset, preferentially at frontal locations. This is in line with the hypothesis 
of top-down modulation of the encoding areas by the frontal executive cortex (Nyhus and Curran, 2010). Parietal and temporal theta oscillation might be related to stimuli processing in associative areas of the cortex and encoding in the structures responsible for the episodic memory (medial temporal lobe). However, the precise sources of activity could not be determined with this low-density array of electrodes. The present study design did not allow to discriminate the effects of novelty expectancy and level of processing over theta oscillations. However, we suggest that both of them should be present in an emotional context and that they will subsequently engage attention and enhance memory formation.

The time window of the N1 potential falls within the peak of theta oscillations increase and overlaps at some locations of the scalp. Interestingly, both theta oscillations and N1 have been related to the same processes (Luck et al., 1990; Klimesch et al., 1997b; Hillyard and Anllo-Vento, 1998; Klimesch, 1999; Bradley et al., 2001; Herrmann and Knight, 2001; Osipova et al., 2006; Beaucousin et al., 2011).

\section{ALPHA OSCILLATIONS}

Alpha power decrease has been extensively studied and consistently related to attention and memory encoding (Klimesch, 1997, 1999; Klimesch et al., 1997b, 2008). This alpha event related desynchronization (ERD) is accompanied by a reset of alpha phase that lead to an increase in the alpha evoked activity. This is called the alpha paradox (Klimesch et al., 1999, 2000; Herrmann and Knight, 2001).

In the extensive work about alpha ERD during memory tasks (preferentially semantic tasks) the called lower alpha (around 6-10 Hz) has been related to expectancy and attention, and showed a widespread distribution over the entire scalp. Note that the results of Theta oscillations $(4-7 \mathrm{~Hz})$ described before are in line with the results of the ERD literature. Meanwhile, upper alpha (around 10-12 Hz) showed a more restricted distribution (central and parietal locations) and has been related to semantic processing (Klimesch et al., 1997a,b, 2005; Klimesch, 1999).

The results of the present study demonstrated that evoked alpha oscillations $(10-12 \mathrm{~Hz})$ recorded at $\mathrm{P} 4$ were higher at the onset of the stimuli embedded in the emotional context. This frequency band is compatible with the called upper alpha. However, the contribution of semantic processing, that is suggested to be related to upper alpha ERD, has not been searched using this test of emotional memory. Anyway, since this is an episodic memory task, the influence of semantic processing should not be relevant. Additionally, since the ERD literature has focused on the semantic memory tasks it is possible that the encoding of the two declarative memory subtypes displays a hemispherespecific pattern.

Although spectral perturbations and EP are obtained by different methodologies, both measures are related and could represent similar, or at least, complementary processes (Klimesch et al., 1999, 2000; Herrmann and Knight, 2001; Makeig et al., 2004). Noteworthy, such changes in the alpha oscillations were restricted to the same location in which the P300 event-related potential was increased during the encoding of the same emotional memory task (Gasbarri et al., 2006).

\section{GAMMA OSCILLATIONS}

Gamma oscillations seem to be involved in almost every class of sensory and cognitive functions (Klimesch et al., 2008). One study found higher induced occipital gamma activity during the encoding (0.3-1 s after stimuli onset) of later remembered pictures (Osipova et al., 2006). Noteworthy, the onset of the pictures in that study was not predictable. With predictable timing, on the other hand, evoked and induced gamma activity increases were related to successful encoding (Sederberg et al., $2003,2007)$. The present study found that evoked (0-0.3 s) and induced $(0.3-0.5 \mathrm{~s})$ gamma activity present in different loci were higher for Phase 2 items. Evoked gamma activity has been related to expectancy and attention (Freunberger et al., 2007). This can be the case of the present study, because all the stimuli had a predictable onset. On the other hand, induced gamma activity is thought to reflect visual information processing (Freunberger et al., 2007). Gamma oscillations, both evoked and induced, showed different patterns of activity between $\mathrm{E}$ and $\mathrm{N}$ groups. As shown by a previous study using common nouns list, the subsequent memory effect can also be related to a decrease of the low gamma activity $(<42 \mathrm{~Hz})$ that coexists with gamma activity increase at other locations (Sederberg et al., 2007). Alternatively, our results might be a consequence of the different context that confers diverse meanings to the same picture. It is reasonable to assume that the oriented attention and the perceived characteristics of the stimuli differed between groups. Those differences might account for the frequency, temporal and spatial differences in the gamma activity.

\section{THETA AND GAMMA INTERACTION}

Theta activity seems to facilitate communication between distant neural assemblies while gamma activity seems to reflect shortdistance synchrony (Buzsaki and Draguhn, 2004; Axmacher et al., 2006; Jutras and Buffalo, 2010; Nyhus and Curran, 2010). Both rhythms are intimately associated and their interaction might create the conditions necessary to long-term hippocampus-dependent memory formation (McCartney et al., 2004; Jutras and Buffalo, 2010). Additionally, the evidence suggests that theta and gamma oscillations interact in order to encode and organize the working memory representations. Extrapolating this concept, it is proposed that this interaction might serve as a working memory buffer for the encoding of episodic memories (Klimesch et al., 2008; Nyhus and Curran, 2010).

However, this hypothesis still waits for conclusive empirical evidence because the areas that showed increased theta and gamma oscillations do not overlap perfectly (Sederberg et al., 2003, 2007; Osipova et al., 2006). Furthermore, to our knowledge, cross-frequency correlation, coherence or another measure of functional connectivity that involve both theta and gamma oscillations have not been explored during the encoding of episodic memory.

Theta and high gamma oscillations were higher during the encoding of Phase 2 stimuli in the emotional context and some areas of the left hemisphere showed increases in both frequency bands at similar time windows. Although functional connectivity was not tested, this partial overlapping suggests the interaction between theta and gamma activities. 
We suggest that the stimuli processing within an emotional context is facilitated by mechanisms of expectancy, attention, and sensory processing that are recruited by the context itself, probably through a top-down control. We conclude that similar mechanisms are responsible for the subsequent memory effect for stimuli with emotional content or context. Despite each measure showed a unique pattern of activity related to the functional specialization of the cortical areas, $\mathrm{N} 1$, theta and gamma activity overlapped at the left prefrontal, frontal, parietal, and temporal locations. This lateralization is in line with the immense majority of the evidence that link the episodic memory encoding with the left hemisphere.

\section{REFERENCES}

Axmacher, N., Mormann, F., Fernandez, G., Elger, C. E., and Fell, J. (2006). Memory formation by neuronal synchronization. Brain Res. Rev. 52, 170-182.

Barrett, L. F., Lindquist, K. A., and Gendron, M. (2007). Language as context for the perception of emotion. Trends Cogn. Sci. 11, 327-332.

Beaucousin, V., Cassotti, M., Simon, G., Pineau, A., Kostova, M., Houde, O., and Poirel, N. (2011). ERP evidence of a meaningfulness impact on visual global/local processing: when meaning captures attention. Neuropsychologia 49, 1258-1266.

Bell, A. J., and Sejnowski, T. J. (1995). An information-maximization approach to blind separation and blind deconvolution. Neural. Comput. 7, 1129-1159.

Bentin, S., and Golland, Y. (2002). Meaningful processing of meaningless stimuli: the influence of perceptual experience on early visual processing of faces. Cognition 86, B1-B14.

Bradley, M. M., Codispoti, M., Cuthbert, B. N., and Lang, P. J. (2001). Emotion and motivation I: defensive and appetitive reactions in picture processing. Emotion 1, 276-298.

Bradley, M. M., and Lang, P. J. (1994). Measuring emotion: the SelfAssessment Manikin and the Semantic Differential. J. Behav. Ther. Exp. Psychiatry. 25, 49-59.

Buzsaki, G., and Draguhn, A. (2004). Neuronal oscillations in cortical networks. Science 304, 1926-1929.

Cahill, L., and McGaugh, J. L. (1995). A novel demonstration of enhanced memory associated with emotional arousal. Conscious. Cogn. 4, 410-421.

Delorme, A., and Makeig, S. (2004). EEGLAB: an open source toolbox for analysis of single-trial EEG dynamics including independent component analysis. J. Neurosci. Methods 134, 9-21.

Dolan, R. J. (2002). Emotion, cognition, and behavior. Science 298, 1191-1194.
Duzel, E., Penny, W. D., and Burgess, N. (2010). Brain oscillations and memory. Curr. Opin. Neurobiol. 20, 143-149.

Foti, D., Hajcak, G., and Dien, J. (2009). Differentiating neural responses to emotional pictures: evidence from temporal-spatial PCA. Psychophysiology 46, 521-530.

Frank, J. E., and Tomaz, C. (2000). Enhancement of declarative memory associated with emotional content in a Brazilian sample. Braz. J. Med. Biol. Res. 33, 1483-1489.

Freunberger, R., Klimesch, W., Sauseng, P., Griesmayr, B., Holler, Y., Pecherstorfer, T., and Hanslmayr, S. (2007). Gamma oscillatory activity in a visual discrimination task. Brain Res. Bull. 71, 593-600.

Gasbarri, A., Arnone, B., Pompili, A., Marchetti, A., Pacitti, F., Calil, S. S., Pacitti, C., Tavares, M. C., and Tomaz, C. (2006). Sex-related lateralized effect of emotional content on declarative memory: an event related potential study. Behav. Brain Res. 168, 177-184.

Gasbarri, A., Pompili, A., Arnone, B., D'Onofrio, A., Marchetti, A., Tavares, M. C., and Tomaz, C. (2005). Declarative memory retention and emotional stimuli. A study of an Italian sample. Funct. Neurol. 20, 157-162.

Gorenstein, C., and Andrade, L. (1996). Validation of a Portuguese version of the Beck Depression Inventory and the State-Trait Anxiety Inventory in Brazilian subjects. Braz. J. Med. Biol. Res. 29, 453-457.

Guderian, S., Schott, B. H., RichardsonKlavehn, A., and Duzel, E. (2009). Medial temporal theta state before an event predicts episodic encoding success in humans. Proc. Natl. Acad. Sci. U.S.A. 106, 5365-5370.

Herrmann, C. S., and Knight, R. T. (2001). Mechanisms of human attention: event-related potentials and oscillations. Neurosci. Biobehav. Rev. 25, 465-476.

Taken together, this work does an important contribution to the characterization of the brain dynamics during the encoding of information in the emotional memory task and, by extension, adds new evidence about the brain processing of relevant environmental stimuli.

\section{ACKNOWLEDGMENTS}

The preparation of this manuscript was supported by a Conselho Nacional de Desenvolvimento Científico e Tecnológico (CNPq) scholarship to Carlos Uribe (140075/2008-9) and by a Fundação de Apóio à Pesquisa do Distrito Federal (FAPDF) grant to Carlos Tomaz (193.000.256/2007).

Hillyard, S.A., and Anllo-Vento, L. (1998). Event-related brain potentials in the study of visual selective attention. Proc. Natl. Acad. Sci. U.S.A. 95 781-787.

Jutras, M. J., and Buffalo, E. A. (2010). Synchronous neural activity and memory formation. Curr. Opin. Neurobiol. 20, 150-155.

Klimesch, W. (1997). EEG-alpha rhythms and memory processes. Int J. Psychophysiol. 26, 319-340.

Klimesch, W. (1999). EEG alpha and theta oscillations reflect cognitive and memory performance: a review and analysis. Brain Res. Brain Res. Rev 29, 169-195.

Klimesch,W., Doppelmayr, M., Pachinger T., and Russegger, H. (1997a). Eventrelated desynchronization in the alpha band and the processing of semantic information. Brain Res. Cogn. Brain Res. 6, 83-94.

Klimesch, W., Doppelmayr, M., Schimke, H., and Ripper, B. (1997b). Theta synchronization and alpha desynchronization in a memory task. Psychophysiology 34, 169-176.

Klimesch, W., Doppelmayr, M., Rohm, D., Pollhuber, D., and Stadler, W. (2000). Simultaneous desynchronization and synchronization of different alpha responses in the human electroencephalograph: a neglected paradox? Neurosci. Lett. 284, 97-100.

Klimesch, W., Doppelmayr, M., Schwaiger, J., Auinger, P., and Winkler, T. (1999). 'Paradoxical' alpha synchronization in a memory task. Brain Res. Cogn. Brain Res. 7, 493-501.

Klimesch, W., Freunberger, R., Sauseng, P. and Gruber,W. (2008). A short review of slow phase synchronization and memory: evidence for control processes in different memory systems? Brain Res. 1235, 31-44.

Klimesch, W., Schack, B., and Sauseng, P. (2005). The functional significance of theta and upper alpha oscillations. Exp. Psychol. 52, 99-108.
LaBar, K. S., and Cabeza, R. (2006) Cognitive neuroscience of emotional memory. Nat. Rev. Neurosci. 7, 54-64.

Luck, S. J., Heinze, H. J., Mangun, G. R., and Hillyard, S.A. (1990). Visual eventrelated potentials index focused attention within bilateral stimulus arrays. II. Functional dissociation of $\mathrm{P} 1$ and $\mathrm{N} 1$ components. Electroencephalogr. Clin. Neurophysiol. 75, 528-542.

Makeig, S., Debener, S., Onton, J., and Delorme, A. (2004). Mining eventrelated brain dynamics. Trends Cogn Sci. 8, 204-210.

McCartney, H., Johnson, A. D., Weil, Z M., and Givens, B. (2004). Theta reset produces optimal conditions for longterm potentiation. Hippocampus 14 684-687.

McGaugh, J. L. (2006). Make mild moments memorable: add a little arousal. Trends Cogn. Sci. 10,345-347.

Nyhus, E., and Curran, T. (2010). Functional role of gamma and theta oscillations in episodic memory. Neurosci. Biobehav. Rev.34, 1023-1035.

Osipova, D., Takashima, A., Oostenveld, R., Fernandez, G., Maris, E., and Jensen, O. (2006). Theta and gamma oscillations predict encoding and retrieval of declarative memory. $J$. Neurosci. 26, 7523-7531.

Pastor, M. C., Bradley, M. M., Low, A., Versace, F., Molto, J., and Lang, P. J. (2008). Affective picture perception: emotion, context, and the late positive potential. Brain Res. 1189, 145-151.

Schupp, H. T., Stockburger, J., Codispoti, M., Junghofer, M., Weike, A. I., and Hamm, A. O. (2007). Selective visual attention to emotion. J. Neurosci. 27, 1082-1089.

Sederberg, P. B., Kahana, M. J., Howard, M. W., Donner, E. J., and Madsen, J. R. (2003). Theta and gamma oscillations during encoding predict subsequent recall. J. Neurosci. 23, 10809-10814.

Sederberg, P. B., Schulze-Bonhage, A., Madsen, J. R., Bromfield, E. B. Mccarthy, D. C., Brandt, A., Tully, M. S., and Kahana, M. J. (2007) 
Hippocampal and neocortical gamma oscillations predict memory formation in humans. Cereb. Cortex 17, 1190-1196.

Töllner, T., Gramann, K., Muller, H. J., and Eimer, M. (2009). The anterior N1 component as an index of modality shifting. J. Cogn. Neurosci. 21, 1653-1669.

Uribe, C., Conde, C., Botelho, S., and Tomaz, C. (2008). Effects of emotionally charged content over behavioral and physiological responses during memory encoding, consolidation and recognition. Neurobiologia 71, 89-98.

Vogel, E. K., and Luck, S. J. (2000). The visual N1 component as an index of a discrimination process. Psychophysiology 37, 190-203.

Weinberg, A., and Hajcak, G. (2010). Beyond good and evil: the time-course of neural activity elicited by specific picture content. Emotion 10,767-782.
Conflict of Interest Statement: The authors declare that the research was conducted in the absence of any commercial or financial relationships that could be construed as a potential conflict of interest.

Received: 14 June 2011; paperpending published: 16 June 2011; accepted: 17 June 2011; published online: 30 June 2011.

Citation: Uribe CE, Garcia A and Tomaz

C (2011) Electroencephalographic brain dynamics of memory encoding in emotionally arousing context. Front. Behav. Neurosci. 5:35. doi: 10.3389/ fnbeh.2011.00035

Copyright (c) 2011 Uribe, Garcia and Tomaz. This is an open-access article subject to a non-exclusive license between the authors and Frontiers Media SA, which permits use, distribution and reproduction in other forums, provided the original authors and source are credited and other Frontiers conditions are complied with. 